CYBERNETICS AND INFORMATION TECHNOLOGIES • Volume 18, No 5

Special Thematic Issue on Optimal Codes and Related Topics

\title{
On Tight Optimal Conflict-Avoiding Codes for 3, 4, 5 and 6 Active Users
}

\author{
Tsonka Baicheva ${ }^{1,2}$, Svetlana Topalova ${ }^{1}$ \\ ${ }^{1}$ Institute of Mathematics and Informatics, Bulgarian Academy of Sciences, 1113 Sofia, Bulgaria \\ ${ }^{2}$ D. A. Tsenov Academy of Economics, 5250 Svishtov, Bulgaria \\ E-mails: tsonka@math.bas.bgrsvetlana@math.bas.bg
}

Abstract: We classify tight optimal conflict-avoiding codes of weights 3, 4, 5 and 6 and given small lengths.

Keywords: Conflict-avoiding codes, tight optimal codes, protocol sequences.

\section{Introduction}

Conflict-Avoiding Codes (CAC) of weight $k$, length $n$ and cardinality $M$ can be applied to avoid collisions in channels with asynchronous multiple access without feedback. In that case the number of codewords $M$ corresponds to the maximum number of users of the channel and the weight $k$ to the maximum number of active users at a given moment. It is assumed that time is partitioned into intervals (slots) and all users have slot synchronization. No other synchronization is assumed. The assigned to each user protocol sequences formed from codewords of a suitable $\mathrm{CAC}$, must allow each of $k$ active users to transmit a data packet successfully in one of $k$ attempts during $n$ time slots without collisions with other active users.

Definition 1. A conflict-avoiding code of length $n$ for $k$ active users $((n, k)$ CAC) is a set $C \subseteq\{(0,1)\}^{n}$ of binary vectors, or codewords, all of Hamming weight $k$, such that arbitrary cyclic shifts $x^{\prime}, y^{\prime}$ of distinct codewords $x, y \in C$ intersect in at most one coordinate, i.e., $\operatorname{dist}\left(x^{\prime}, y^{\prime}\right) \geq 2 k-2$, where $\operatorname{dist}\left(x^{\prime}, y^{\prime}\right)$ is the Hamming distance between $x^{\prime}$ and $y^{\prime}$.

Definition 2. The support $\operatorname{supp}(x)$ of a codeword $x$ is the set of indices of its nonzero coordinates.

It is more convenient for our investigation to use $\operatorname{supp}(x)$ instead of $x$. Denote $\operatorname{supp}(x)$ by $X$ and let $X=\left\{x_{0}, x_{1}, \ldots, x_{k-1}\right\}$. The support of a cyclic shift of $x$ is then a translate $\left\{x_{0}+t, x_{1}+t, \ldots, x_{k-1}+t\right\}$ of $X$, where addition is modulo $n$. 
Denote by $\Delta^{\prime}(X)=\left\{x_{i}-x_{j}(\bmod n) \mid x_{i}, x_{j} \in X, i \neq j\right\}$ the multiset of differences of $X$ and by $\Delta(X)$ its corresponding set.

Definition 3. The number $|\Delta(X)|$ is called the type of $X$ and denoted by $T(X)$.

Definition 4. Any $(n, k)$ conflict-avoiding code $C$ can be considered as a collection of $k$ subsets of $Z_{n}$ such that

$$
\Delta(X) \cap \Delta(Y)=\varnothing \text { for any } X, Y \in C \text {. }
$$

Therefore when we talk of codewords below, we will actually mean $k$ subsets of $Z_{n}$.

Definition 5. Two codewords are equivalent if $\Delta(X)=\Delta(Y)$.

We assume that $x_{0}<x_{1}<\ldots<x_{k-1}$ for each codeword $X=\left\{x_{0}, x_{1}, \ldots, x_{k-1}\right\}$. We define a lexicographic order on the codewords in the following way.

Definition 6. The codeword $X^{\prime}=\left\{x_{0}^{\prime}, x_{1}^{\prime}, \ldots, x_{k-1}^{\prime}\right\}$ is lexicographically smaller than $X^{\prime \prime}=\left\{x^{\prime \prime}{ }_{0}, x^{\prime \prime}{ }_{1}, \ldots, x^{\prime \prime}{ }_{k-1}\right\}$ if $T\left(X^{\prime}\right)<T\left(X^{\prime \prime}\right)$, or if $\left|\Delta\left(X^{\prime}\right)\right|=\left|\Delta\left(X^{\prime \prime}\right)\right|$ and $x_{i}^{\prime}=x^{\prime \prime}{ }_{i}$ for $i<j$, but $x_{j}^{\prime}<x^{\prime \prime}{ }_{j}$ for some $j$.

Without loss of generality we assume that each codeword is lexicographically smaller than its translates. This means that $x_{0}=0$ for each codeword and when we say that $X_{1}$ is mapped to $X_{2}$ by the permutation $\varphi$, we mean that $X_{2}$ is the smallest translate of $\varphi\left(X_{1}\right)$.

Definition 7. An $(n, k)$ CAC of size $s$ is tight (perfect) if $\bigcup_{i=1}^{s}\left|\Delta X_{i}\right|=n-1$, that is if all nonzero differences are covered.

Example 1. The four codewords of a tight $(15,3)$ CAC are listed below together with their sets of differences and types:

$$
\begin{array}{lll}
X_{1}=\{0,5,10\} & \Delta\left(X_{1}\right)=\{5,10\} & \mathrm{T}\left(X_{1}\right)=2 \\
X_{2}=\{0,1,2\} & \Delta\left(X_{2}\right)=\{1,2,13,14\} & \mathrm{T}\left(X_{2}\right)=4 \\
X_{3}=\{0,7,11\} & \Delta\left(X_{3}\right)=\{4,7,8,11\} & \mathrm{T}\left(X_{3}\right)=4 \\
X_{4}=\{0,6,12\} & \Delta\left(X_{4}\right)=\{3,6,9,12\} & \mathrm{T}\left(X_{4}\right)=4 .
\end{array}
$$

Definition 8. Denote by $M(n, k)$ the maximum cardinality of a CAC of length $n$. A conflict-avoiding code $C$ is said to be optimal if $|C|=M(n, k)$.

The advantage of using optimal codes is that they enable the largest number of asynchronous users to transmit packets efficiently and reliably through a multipleaccess channel without feedback.

Definition 9. Two $(n, k) \mathrm{CACs}$ are multiplier equivalent if they can be obtained from one another by a multiplier automorphism of $Z_{n}$ and replacement of codewords by some of their translates.

Remark. Any CAC of length $n$ for $k$ active users can be viewed as an $(n, k, k, 1)$ optical orthogonal code. Such codes are used in optical code-division multiple access channels [1].

Optimal CACs as protocol sequences for a multiple-access collision channel without feedback have been studied in many works [2-18]. The case with three active users $(k=3)$ is completely settled. Several optimal constructions for weights 4 and 5 can be found in [14]. General upper bounds on the size of constant weight CACs applicable to all code lengths and all Hamming weights are derived in [15] and [16]. Examples of small length CACs can be found in [17], and classification results about CACs of weights up to 7 and small lengths in [2] and [3]. 
Tight CACs have additional properties which make them interesting as incidence structures and thus might be more appropriate in constructions of other codes or combinatorial structures. There are, for instance, recursive constructions of CACs from tight CACs of smaller lengths [11]. That is why tight CACs are of particular importance.

Some existence conditions and constructions of tight $(n, 3)$ CACs for definite values of $n$ can be found in $[13,5,18,10,11]$, but checking the conditions and applying the constructions is often a difficult job for those who are interested in such codes from any application point of view. There are tight CACs among those which are classified in [2,3], but they are not explicitly listed. That is why we think that the online availability of the nonequivalent tight CACs of small $k$ and $n$ will be useful for any application purposes. This motivated us to present the current classification of tight optimal CACs.

Our investigation concerns optimal tight CACs of weight $k=3$ and length $n \leq 111, k=4$ and $n \leq 120, k=5$ and $n \leq 118, k=6$ and $n \leq 119$, and $k=7$ and $n \leq 95$. The construction algorithm which we use here, allows us to classify the tight codes for 21 lengths for which the optimal CACs are not classified in [2, 3].

\section{Algorithm}

Our algorithm constructs tight codes with a given cardinality $s$. It performs backtrack search on the set of all possible codewords. We obtain them in advance from all nonequivalent (by Definition 5) $k$-sets of $Z_{n}$. We find the type of the codewords defined by these $k$-sets. Let $T_{\min }$ be the smallest type. We want to construct a code of $s$ codewords. Such a code cannot have codewords of a type greater than $T_{\max }=n-1-(s-1) T_{\min }$. Possible codewords are only $k$-sets of type at most $T_{\max }$. Therefore the set of possible codewords for a code of cardinality $s+1$ might be much smaller than that of a code of cardinality $s$.

The possible codewords are partitioned in groups, such that each multiplier automorphism of $Z_{n}$ maps any codeword to a codeword of the same group. We call leader the lexicographically smallest codeword of the group. We sort the groups with respect to the lexicographic order of their leaders and save them. For each possible codeword we know the possible codeword to which it is mapped by any automorphism of $Z_{n}$, and this makes the below described minimality test very fast.

The set of possible codewords we construct here differs from the similar sets used in [2] and [3]. In [2] equivalence of codewords is not defined, while here we give Definition 5 following [18]. By this definition we filter away possible codewords which might lead to codes which are different as combinatorial structures, but which perform the same as CACs for channels with asynchronous multiple access without feedback. The smaller search set makes the classification algorithm much faster. In [3] we remove a group from the set of possible codewords if the set of differences of its leader is the same as the set of differences of another leader, while here we remove a group from the set of possible codewords if the set of differences of its leader is the same as the set of differences of any possible codeword from another group. 
The backtrack search on the possible codewords is similar to the one we used in [2] and [3], namely when we add the next codeword, we speed up the algorithm by performing a Minimality test and a Type test to the current partial solution. Apart from this, to construct tight codes here, we apply a Tight test too. We briefly describe the three tests.

Suppose that $r$ codewords of the code have been already found. Let $T$ be the type of the $r$-th codeword, and let $d$ be the number of distinct differences covered by the $r$ codewords.

Type test. We only look for codes with a definite number $s$ of codewords. That is why the type of the remaining possible codewords (of the array we choose them from) is at least as big as that of the $r$-th chosen one. That is why $d+(s-r) T \leq n-1$. If this does not hold, the next possibility for the $(r-1)$-st codeword is considered.

Tight test. Knowing the types of the remaining possible codewords, we try to find codeword types $T_{1}, \ldots, T_{s-r}$ such that $d+T_{1}+\ldots+T_{s-r}=n-1$. If this does not hold, the next possibility for the $(r-1)$-st codeword is considered.

Minimality test. We check if the current partial solution can be mapped to a lexicographically smaller one by some of the automorphisms of $Z_{n}$. If it can, an equivalent partial solution has already been considered, and we look for the next possibility for the current codeword.

\section{Results}

We consider values of $n$ for which the cardinality of the optimal CACs is known $[2,3]$. The results are summarized in the tables below. Only tight CACs with at least 2 codewords are included. Lengths for which no tight optimal CAC exists, are not presented. The number of nonequivalent tight optimal CACs is given in column TCACs. One can see that this number is very big if the size of the optimal codes is relatively small for the given length. Tight optimal codes with relatively big sizes for the given length range are most interesting and usually there are not many of them.

All the constructed codes are available online and can be downloaded from http://www.moi.math.bas.bg/ svetlana. Information on the different types of codes (with respect to the types of the codewords) is also given there as illustrated in the following example.

Example 2. There are nine nonequivalent $(119,6)$ tight optimal CACs of four code-types which are presented as:

$\begin{array}{lllll}\text { 0) } 2: & 6-1 & 10-5 & 12-3 & 26-1 \\ \text { 1) } 2: & 6-1 & 10-4 & 12-4 & 24-1 \\ \text { 2) } 1: & 6-1 & 12-8 & 16-1 & \\ \text { 3) } 4: & 6-1 & 12-7 & 14-2 & \end{array}$

This means that there are two codes of code-type 0 (with one codeword of type 6 , five codewords of type 10 , three of type 12 and one of type 26), two codes of code-type 1 , one of code-type 2 and four of code-type 3. 
Table 1. Tight optimal CACs with $k=3$ and $n \leq 111$

\begin{tabular}{|c|c|c|c|c|c|}
\hline$n$ & $M(n, 3)$ & TCACs & $n$ & $M(n, 3)$ & TCACs \\
\hline 13 & 3 & 1 & 47 & 11 & 1 \\
\hline 15 & 4 & 1 & 48 & 10 & 1,602 \\
\hline 16 & 3 & 2 & 49 & 11 & 22 \\
\hline 17 & 4 & 1 & 51 & 13 & 4 \\
\hline 18 & 4 & 2 & 52 & 11 & 621 \\
\hline 19 & 4 & 2 & 53 & 13 & 1 \\
\hline 20 & 4 & 3 & 54 & 13 & 2 \\
\hline 21 & 4 & 5 & 56 & 12 & 170 \\
\hline 23 & 5 & 1 & 57 & 14 & 5 \\
\hline 24 & 5 & 8 & 59 & 14 & 2 \\
\hline 25 & 6 & 1 & 60 & 13 & 7,702 \\
\hline 27 & 6 & 2 & 61 & 15 & 1 \\
\hline 28 & 6 & 5 & 63 & 15 & 46 \\
\hline 29 & 7 & 1 & 64 & 13 & 101,136 \\
\hline 30 & 7 & 2 & 65 & 16 & 6 \\
\hline 31 & 7 & 2 & 66 & 16 & 1 \\
\hline 32 & 7 & 2 & 67 & 16 & 4 \\
\hline 33 & 8 & 3 & 68 & 15 & 200 \\
\hline 35 & 8 & 1 & 69 & 17 & 7 \\
\hline 36 & 8 & 30 & 71 & 17 & 3 \\
\hline 37 & 9 & 1 & 72 & 16 & 1,333 \\
\hline 39 & 10 & 2 & 73 & 17 & 80 \\
\hline 40 & 8 & 195 & 75 & 19 & 4 \\
\hline 41 & 10 & 1 & 76 & 16 & 377,203 \\
\hline 42 & 10 & 1 & 77 & 18 & 78 \\
\hline 43 & 10 & 2 & 78 & 19 & 1 \\
\hline 44 & 9 & 308 & 79 & 19 & 5 \\
\hline
\end{tabular}

\begin{tabular}{|c|c|c|}
\hline$n$ & $M(n, 3)$ & TCACs \\
\hline 80 & 17 & 209,575 \\
\hline 81 & 19 & 1,758 \\
\hline 83 & 20 & 4 \\
\hline 84 & 19 & 2,464 \\
\hline 85 & 21 & 10 \\
\hline 87 & 22 & 2 \\
\hline 88 & 19 & 39,552 \\
\hline 89 & 21 & 125 \\
\hline 90 & 22 & 3 \\
\hline 91 & 22 & 4 \\
\hline 92 & 20 & 200,224 \\
\hline 93 & 23 & 6 \\
\hline 95 & 23 & 2 \\
\hline 96 & 21 & $3,411,597$ \\
\hline 97 & 24 & 1 \\
\hline 99 & 24 & 40 \\
\hline 100 & 22 & 40,928 \\
\hline 101 & 25 & 1 \\
\hline 102 & 25 & 1 \\
\hline 103 & 25 & 5 \\
\hline 104 & 22 & $\geq 5,000,000$ \\
\hline 105 & 26 & 22 \\
\hline 107 & 26 & 2 \\
\hline 108 & 24 & $\geq 3,600,000$ \\
\hline 109 & 27 & 2 \\
\hline 111 & 28 & 2 \\
\hline & & \\
\hline
\end{tabular}

Table 2. Tight optimal CACs with $k=4$ and $n \leq 120$

\begin{tabular}{|c|c|c|}
\hline$n$ & $M(n, 4)$ & TCACs \\
\hline 17 & 2 & 1 \\
\hline 20 & 3 & 2 \\
\hline 24 & 3 & 2 \\
\hline 25 & 3 & 1 \\
\hline 28 & 4 & 4 \\
\hline 30 & 4 & 2 \\
\hline 32 & 5 & 1 \\
\hline 34 & 5 & 1 \\
\hline 35 & 6 & 1 \\
\hline 36 & 5 & 13 \\
\hline 37 & 6 & 1 \\
\hline 38 & 6 & 2 \\
\hline 39 & 5 & 1 \\
\hline 40 & 6 & 24 \\
\hline 41 & 6 & 2 \\
\hline 42 & 6 & 3 \\
\hline 43 & 6 & 11 \\
\hline 44 & 7 & 6 \\
\hline 45 & 6 & 66 \\
\hline 46 & 7 & 6 \\
\hline 47 & 7 & 1 \\
\hline
\end{tabular}

\begin{tabular}{|c|c|c|}
\hline$n$ & $M(n, 4)$ & TCACs \\
\hline 57 & 8 & 3 \\
\hline 58 & 9 & 1 \\
\hline 59 & 9 & 1 \\
\hline 60 & 9 & 57 \\
\hline 61 & 9 & 4 \\
\hline 62 & 9 & 302 \\
\hline 63 & 9 & 2 \\
\hline 64 & 10 & 6 \\
\hline 65 & 10 & 5 \\
\hline 67 & 10 & 4 \\
\hline 68 & 10 & 629 \\
\hline 69 & 11 & 0 \\
\hline 70 & 11 & 9 \\
\hline 71 & 10 & 440 \\
\hline 72 & 11 & 11 \\
\hline 73 & 11 & 3 \\
\hline 74 & 11 & 294 \\
\hline 75 & 11 & 38 \\
\hline 76 & 12 & 5 \\
\hline 77 & 12 & 1 \\
\hline 78 & 11 & 26,106 \\
\hline
\end{tabular}

\begin{tabular}{|c|c|c|}
\hline$n$ & $M(n, 4)$ & TCACs \\
\hline 88 & 14 & 4 \\
\hline 89 & 13 & 517 \\
\hline 90 & 14 & 8 \\
\hline 91 & 14 & 1 \\
\hline 92 & 14 & 572 \\
\hline 94 & 14 & 3,827 \\
\hline 95 & 14 & 4,386 \\
\hline 96 & 14 & 22,577 \\
\hline 97 & 15 & 2 \\
\hline 98 & 15 & 72 \\
\hline 99 & 14 & 160,321 \\
\hline 100 & 16 & 21 \\
\hline 101 & 15 & 337 \\
\hline 102 & 15 & 17,812 \\
\hline 104 & 17 & 2 \\
\hline 105 & 16 & 11 \\
\hline 106 & 16 & 2,950 \\
\hline 107 & 16 & 245 \\
\hline 108 & 16 & 10,812 \\
\hline 109 & 16 & 6,676 \\
\hline 110 & 17 & 692 \\
\hline
\end{tabular}


Table 2 (c o n t i n u e d)

\begin{tabular}{|c|c|c|}
\hline$n$ & $M(n, 4)$ & TCACs \\
\hline 48 & 7 & 24 \\
\hline 49 & 8 & 1 \\
\hline 50 & 7 & 350 \\
\hline 51 & 7 & 4 \\
\hline 52 & 8 & 18 \\
\hline 53 & 7 & 225 \\
\hline 55 & 8 & 37 \\
\hline 56 & 8 & 310 \\
\hline
\end{tabular}

\begin{tabular}{|c|c|c|}
\hline$n$ & $M(n, 4)$ & TCACs \\
\hline 79 & 12 & 3 \\
\hline 80 & 13 & 2 \\
\hline 82 & 12 & 3,797 \\
\hline 83 & 12 & 470 \\
\hline 84 & 13 & 1 \\
\hline 85 & 13 & 57 \\
\hline 86 & 13 & 206 \\
\hline 87 & 13 & 2 \\
\hline
\end{tabular}

\begin{tabular}{|c|c|c|}
\hline$n$ & $M(n, 4)$ & TCACs \\
\hline 112 & 17 & 4,858 \\
\hline 113 & 17 & 231 \\
\hline 114 & 17 & 9,480 \\
\hline 115 & 17 & 95,927 \\
\hline 116 & 18 & 340 \\
\hline 117 & 17 & 162,282 \\
\hline 118 & 18 & 2,455 \\
\hline 120 & 18 & 45,890 \\
\hline
\end{tabular}

Table 3. Tight optimal CACs with $k=5$ and $n \leq 118$

\begin{tabular}{|c|c|c|}
\hline$n$ & $M(n, 5)$ & TCACs \\
\hline 43 & 3 & 1 \\
\hline 45 & 6 & 1 \\
\hline 47 & 5 & 1 \\
\hline 50 & 6 & 1 \\
\hline 54 & 6 & 1 \\
\hline 55 & 6 & 7 \\
\hline 56 & 6 & 2 \\
\hline 60 & 6 & 23 \\
\hline 61 & 6 & 10 \\
\hline 62 & 6 & 5 \\
\hline 63 & 8 & 2 \\
\hline 64 & 6 & 15 \\
\hline 65 & 7 & 11 \\
\hline 66 & 7 & 4 \\
\hline 67 & 7 & 2 \\
\hline 69 & 8 & 1 \\
\hline 70 & 8 & 8 \\
\hline 71 & 7 & 13 \\
\hline 72 & 7 & 64 \\
\hline
\end{tabular}

\begin{tabular}{|c|c|c|}
\hline$n$ & $M(n, 5)$ & TCACs \\
\hline 73 & 8 & 15 \\
\hline 75 & 8 & 34 \\
\hline 77 & 8 & 362 \\
\hline 78 & 8 & 47 \\
\hline 79 & 8 & 19 \\
\hline 80 & 8 & 59 \\
\hline 81 & 9 & 4 \\
\hline 82 & 8 & 39 \\
\hline 83 & 8 & 285 \\
\hline 84 & 8 & 1,302 \\
\hline 85 & 9 & 88 \\
\hline 86 & 9 & 1 \\
\hline 88 & 9 & 2 \\
\hline 89 & 9 & 84 \\
\hline 91 & 10 & 6 \\
\hline 93 & 10 & 9 \\
\hline 95 & 11 & 13 \\
\hline 96 & 9 & 4,214 \\
\hline 97 & 12 & 2 \\
\hline
\end{tabular}

\begin{tabular}{|c|c|c|}
\hline$n$ & $M(n, 5)$ & TCACs \\
\hline 98 & 10 & 25 \\
\hline 99 & 12 & 2 \\
\hline 101 & 10 & 468 \\
\hline 102 & 10 & 1,942 \\
\hline 103 & 10 & 2,104 \\
\hline 104 & 10 & 2,028 \\
\hline 105 & 11 & 1,521 \\
\hline 107 & 11 & 67 \\
\hline 108 & 11 & 58 \\
\hline 109 & 11 & 372 \\
\hline 110 & 12 & 18 \\
\hline 111 & 12 & 94 \\
\hline 112 & 11 & 1,064 \\
\hline 113 & 12 & 6 \\
\hline 114 & 12 & 13 \\
\hline 115 & 13 & 44 \\
\hline 117 & 14 & 2 \\
\hline 118 & 12 & 239 \\
\hline
\end{tabular}

Table 4. Tight optimal CACs with $k=6$ and $n \leq 119$

\begin{tabular}{|c|c|c|}
\hline$n$ & $M(n, 6)$ & TCACs \\
\hline 42 & 3 & 1 \\
\hline 62 & 4 & 1 \\
\hline 66 & 5 & 1 \\
\hline 69 & 5 & 1 \\
\hline 72 & 5 & 1 \\
\hline 77 & 8 & 1 \\
\hline 84 & 8 & 5 \\
\hline 88 & 7 & 2 \\
\hline 89 & 6 & 2 \\
\hline
\end{tabular}

\begin{tabular}{|c|c|c|}
\hline$n$ & $M(n, 6)$ & TCACs \\
\hline 90 & 7 & 1 \\
\hline 91 & 8 & 20 \\
\hline 94 & 8 & 1 \\
\hline 96 & 8 & 2 \\
\hline 98 & 8 & 45 \\
\hline 99 & 8 & 9 \\
\hline 100 & 8 & 3 \\
\hline 103 & 8 & 4 \\
\hline 104 & 8 & 13 \\
\hline
\end{tabular}

\begin{tabular}{|c|c|c|}
\hline$n$ & $M(n, 6)$ & TCACs \\
\hline 107 & 8 & 38 \\
\hline 108 & 9 & 2 \\
\hline 111 & 9 & 1 \\
\hline 112 & 9 & 6 \\
\hline 116 & 9 & 4 \\
\hline 117 & 9 & 60 \\
\hline 119 & 10 & 9 \\
\hline
\end{tabular}

Table 5. Tight optimal CACs with $k=7$ and $n \leq 95$

\begin{tabular}{|c|c|c|}
\hline$n$ & $M(n, 7)$ & TCACs \\
\hline 60 & 2 & 1 \\
\hline 63 & 4 & 2 \\
\hline 91 & 8 & 1 \\
\hline
\end{tabular}


Acknowledgements: The research of the first author was partially supported by the Bulgarian National Science Fund, Contract No DH 12/8, 15.12.2017. The research of the second author was partially supported by the Bulgarian National Science Fund, Contract No DH 02/2, 13.12.2016.

\section{References}

1. C h u n g, F. R. K., J. A. S a 1 e h i, V. K. W e i. Optical Orthogonal Codes: Design, Analysis, and Applications. - IEEE Trans. Inform. Theory, Vol. 35, 1989, No 3, pp. 595-604.

2. B a i c h e v a, T., S. T o p a 1 o v a. Optimal Conflict-Avoiding Codes for 3, 4 and 5 Active Users. - Problems of Information Transmission, Vol. 53, 2017, No 1, pp. 42-50.

3. B a i c h e v a, T., S. T o p a 1 o v a. Classification of Optimal Conflict-Avoiding Codes of Weights 6 and 7. - Electronic Notes in Discrete Mathematics, Vol. 57, March 2017, pp. 9-14.

4. F u, H., Y. L i n, M. M i s h i m a. Optimal Conflict-Avoiding Codes of Even Length and Weight 3. - IEEE Trans. Inform. Theory, Vol. 56, 2010, No 11, pp. 5747-5756.

5. F u, H., Y. L o, K. S h u m. Optimal Conflict-Avoiding Codes of Odd Length and Weight Three.Des. Codes Cryptogr., Vol. 72, 2014, No 2, pp. 289-309.

6. J i mbo, M., M. M i s h i m a, S. J a n is z e w s ki, A. Y. T e y moria n, V. D. Ton che v. On Conflict-Avoiding Codes of Length $n=4 m$ for Three Active Users. - IEEE Trans. Inform. Theory, Vol. 53, 2007, No 8, pp. 2732-2742.

7. L e v e n s h t e i n, V. I. Conflict-Avoiding Codes for Many Active Users. - In: Abstarcts of 14th Internat. Conference Problems of Theoretic Cybernetics, Penza, 2005, pp. 86-86 (in Russian).

8. L e v e n s h t e i n, V. I., V. D. T o n c h e v. Optimal Conflict-Avoiding Codes for Three Active Users. - In: Proc. of IEEE Internat. Symposium on Inform. Theory, Adelaide, 2005, pp. 535-537.

9. L e ve n s h t e i n, V. I. Conflict-Avoiding Codes and Cyclic Triple Systems. - Probl. of Inform. Transm., Vol. 43, 2007, No 3, pp. 199-212.

10. L i n, Y., M. M i s h i m a, J. S a t o h, M. J i m b o. Optimal Equi-Difference Conflict-Avoiding Codes of Odd Length and Weight Three. - Finite Fields Appl., Vol. 26, 2014, pp. 49-68.

11. $\mathrm{M}$ a, W., C. Z h a o, D. S h e n. New Optimal Constructions of Conflict-Avoiding Codes of Odd Length and Weight 3. - Des. Codes Cryptogr., Vol. 73, 2014, No 3, pp. 791-804.

12. M i s h i m a, M., H. F u, S. U r u n o. Optimal Conflict-Avoiding Codes of Length $n=0(\bmod 16)$ and Weight 3. - Des. Codes Cryptogr., Vol. 52, 2009, No 3, pp. 275-291.

13. M o m i h a r a, K. Necessary and Sufficient Conditions for Tight Equi-Difference Conflict Avoiding Codes of Weight 3. - Des. Codes Cryptogr, Vol. 45, 2007, No 3, pp. 379-390.

14. M o m i h a r a, K., M. M ü 1 e r, J. S a t o n, M. J i m b o. Constant Weight Conflict-Avoiding Codes. - SIAM J. Discr. Math., Vol. 21, 2007, No 4, pp. 959-979.

15. S h u m, K. W., W. S. W o n g. A Tight Asymptotic Bound on the Size of Constant-Weight Conflict-Avoiding Codes. - Des. Codes Cryptogr., Vol. 57, 2010, No 1, pp. 1-14.

16. S h u m, K. W., W. S. W o n g, C. S. C h e n. A General Upper Bound on the Size of ConstantWeight Conflict Avoiding Codes. - IEEE Trans. Inform. Theory, Vol. 56, 2010, No 7, pp. 3265-3276.

17. T o n c h e v, V. D. Tables of Conflict-Avoiding Codes. http://www.math.mtu.edu/tonchev/CAC.html

18. W u, S. L., H. L. F u. Optimal Tight Equi-Difference Conflict-Avoiding Codes of Length $n=2^{k} \pm 1$ and Weight 3. - J. Comb. Des., Vol. 21, 2013, No 6, pp. 223-231. 